\title{
Long-term oral administration of sweet chestnut (Castanea sativa Mill.) extract does not affect the contraction ability of isolated ileum
}

\author{
Andrej Javornik, Kaja Blažič, Nika Šehić, Tomaž Snoj \\ University of Ljubljana, Veterinary Faculty, Ljubljana, Slovenia
}

Received July 19, 2018

Accepted April 23, 2019

\begin{abstract}
Extracts of sweet chestnut (Castanea sativa Mill.) which contain hydrolysable tannins are used to prevent diarrhoea in farm animals due to the astringent effect of tannins. The aim of this study was to establish whether long-term treatment with the chestnut extract as a feed additive affects the acetylcholine (ACh)-triggered contractions of isolated rat ileum. Wistar rats of both sexes were divided into 4 male and 4 female groups with 6 animals in each group. The first, second and third groups of both sexes received feed with 3,15 and $30 \mathrm{~g}$ of chestnut extract per $\mathrm{kg}$, respectively. The fourth group of both sexes received feed without the added chestnut extract and served as the control group. After the 90-day trial, the animals were euthanized, and ilea were isolated and placed in an organ bath filled with Tyrode's solution. Ilea were connected to an isometric transducer. Ileal contractions were triggered by $0.005,0.01,0.05,0.1,0.5,1.0,5.0$ and $10.0 \mathrm{mg}$ of ACh, and contractions were recorded. The groups exhibited dose responsiveness to the ACh treatment steps; however, no significant differences in the contraction intensity were observed among the groups at the same ACh dose level. These results suggest that long-term treatment with chestnut extract as an astringent does not influence the intensity of ileal contractions triggered by ACh. Thus, no adverse effect on intestinal motility can be expected during a long-term use of chestnut extract as a feed additive in livestock production; however, further studies in target animal species are needed to confirm the findings.
\end{abstract}

Hydrolysable tannins, intestine contractions, rats

In the livestock industry, sweet chestnut (Castanea sativa Mill.) extract is used as a feed additive because it increases feed conversion, mitigates methane synthesis, increases protein digestibility in ruminants and prevents gastrointestinal infections. These beneficial properties of the chestnut extract are associated with the hydrolysable tannin extract contents (Budriesi et al. 2010). Some tannins exhibit also astringent, antispasmodic, anthelmintic, antimicrobial, antioxidant and anti-inflammatory activities (Huang et al. 2018), while the chemical structure and biological properties of tannins vary between plant species (Aldred 2009; Budriesi et al. 2010; Piluzza et al. 2013).

During ingestion, tannins precipitate proteins on the surface of intestinal mucosa and thus form stable complexes that act as a protective barrier, preventing infections of intestinal mucosa and water loss through the intestinal wall. By this mechanism, tannins exhibit an antidiarrhoeal effect (Aldred 2009; Namkung et al. 2010). Additionally, plant extracts that contain tannins reduce intestinal motility and exert antispasmodic activity (Budriesi et al. 2010; Allem and Janbaz 2018). Budriesi et al. (2010) described the antispasmodic activity of chestnut extracts under in vitro conditions and concluded that the observed antispasmodic mechanism was probably due to an unspecific cellular pathway. Allem and Janbaz (2018) demonstrated that the antispasmodic activity of tannins is most likely due to the modulation of cholinergic receptors and calcium channels in the intestinal wall. Moreover, the antidiarrhoeal and antispasmodic effects of extracts that contain tannins are associated with inhibition of calcium-activated chlorine channels, which have been implicated in many physiological functions such as transepithelial fluid secretion and

Address for correspondence:

Tomaž Snoj

Veterinary Faculty

University of Ljubljana

Phone: +38614779 130

Gerbičeva 60, 1000 Ljubljana, Slovenia 
smooth muscle contraction. Inhibition of calcium-activated chlorine channels is a possible molecular basis for the health benefits of some tannins (Namkung et al. 2010).

In this study, we wanted to establish whether long-term treatment with chestnut extract as a feed additive affects the acetylcholine (ACh)-triggered contraction ability of isolated rat ileum.

\section{Materials and Methods}

The use of animals in the experiment was approved by The Administration of the Republic of Slovenia for Food Safety, Veterinary and Plant Protection, under license No. U34401-27/2017/8.

A total of 48 Wistar rats (24 males and 24 females) at the age of 2 months were divided into 8 (4 male and 4 female) groups with 6 animals in each. The animals were housed under standard laboratory conditions with controlled temperature $\left(22 \pm 3^{\circ} \mathrm{C}\right)$, relative humidity of $30-70 \%$, and 12 -h light/dark cycles. They had ad libitum access to feed and water. For 90 days (13 weeks), 3 male and 3 female experimental groups were fed with feed containing different amounts of chestnut extract (Farmatan, Tanin Sevnica d.d., Sevnica, Slovenia). As declared by the manufacturer, the chestnut extract contains $>71 \%$ of tannins. The first male and female groups (labelled group M 3 and group F 3 , respectively) received feed containing $3 \mathrm{~g}$ of chestnut extract per $\mathrm{kg}$ of feed. The second set of groups of both sexes (M 15 and F 15) received feed with $15 \mathrm{~g}$ chestnut extracts per kg of feed, and the third set of groups of both sexes (M 30 and F 30) received feed with $30 \mathrm{~g}$ chestnut extract per $\mathrm{kg}$ of feed. The fourth set of groups ( $\mathrm{M} \mathrm{C}$ and F C) received feed without the chestnut extract and served as controls. The used chestnut extract contained $75 \%$ hydrolysable tannins. During the trial, feed consumption was determined by weighing the feed before offering it to the animals and then weighing the unconsumed feed, which was performed weekly during cage cleaning. Body weight was also determined weekly (data not shown). Considering the body weight and feed consumption, animals were exposed to the chestnut extract at the doses presented in Table 1.

Table 1. Chestnut extract doses (g/kg b.w./day) for the experimental groups during the 13-week trial.

\begin{tabular}{|c|c|c|c|c|c|c|c|c|c|c|c|c|c|}
\hline \multicolumn{14}{|c|}{ Week of treatment } \\
\hline & 1 & 2 & 3 & 4 & 5 & 6 & 7 & 8 & 9 & 10 & 11 & 12 & 13 \\
\hline Group & \multicolumn{13}{|c|}{ Dose of chestnut extract (g/kg b.w./day) } \\
\hline M 3 & 0.23 & 0.24 & 0.22 & 0.22 & 0.19 & 0.18 & 0.18 & 0.17 & 0.17 & 0.16 & 0.16 & 0.15 & 0.15 \\
\hline M 15 & 1.16 & 1.29 & 1.13 & 1.04 & 0.98 & 0.89 & 0.84 & 0.89 & 0.88 & 0.83 & 0.84 & 0.78 & 0.74 \\
\hline M 30 & 2.44 & 2.50 & 2.25 & 2.17 & 1.99 & 1.89 & 1.88 & 1.77 & 1.77 & 1.69 & 1.68 & 1.60 & 1.54 \\
\hline F3 & 0.27 & 0.23 & 0.23 & 0.23 & 0.21 & 0.19 & 0.20 & 0.20 & 0.19 & 0.19 & 0.19 & 0.19 & 0.18 \\
\hline F 15 & 1.37 & 1.18 & 1.19 & 1.23 & 1.11 & 0.98 & 0.99 & 1.17 & 0.97 & 0.93 & 0.92 & 0.99 & 0.93 \\
\hline F 30 & 2.69 & 2.63 & 2.38 & 2.42 & 2.18 & 2.02 & 2.17 & 2.24 & 2.27 & 2.15 & 1.97 & 2.09 & 2.02 \\
\hline
\end{tabular}

On the $90^{\text {th }}$ day of the trial, animals were euthanized with $\mathrm{CO}_{2}$ and bleeding. Immediately after euthanasia, the abdomen was opened, and a $2 \mathrm{~cm}$ long segment of ileum was excised, flushed and placed into Tyrode's solution (mM): $\mathrm{NaCl} 136.9, \mathrm{KCl} 2.7, \mathrm{CaCl}_{2} 1.8, \mathrm{MgCl}_{2}$ 1.0, $\mathrm{NaHCO}_{3}$ 11.9, $\mathrm{NaH}_{2} \mathrm{PO}_{4} 0.4$, glucose 5.0.

Each isolated ileum was placed vertically in a $20 \mathrm{ml}$ organ bath filled with bubbled $\left(95 \% \mathrm{O}_{2}\right.$ and $\left.5 \% \mathrm{CO}_{2}\right)$ Tyrode's solution at $37^{\circ} \mathrm{C}$. One end of the strip was fixed to the bath bottom, and the other end of the preparation was tied to an isometric transducer (Elunit, Belgrade, Serbia). The initial tension of mounted ileum was set at $1 \mathrm{~g}$. The preparations were allowed to equilibrate for $5 \mathrm{~min}$ under the initial tension. After the equilibration period, ileum of each animal was successively stimulated with the administration of eight different doses $(0.005,0.01$, $0.05,0.1,0.5,1.0,5.0$ or $10.0 \mathrm{mg}$ ) of acetylcholine (ACh) (Sigma-Aldrich, St. Louis, USA), which were injected into the $20 \mathrm{ml}$ organ bath. After each ACh administration, ileum contraction for a given ACh dose was recorded, and ileum was flushed twice with Tyrode's solution before the next ACh administration.

The isometric ileal contractions were determined using SmartPlus 150 software (Elunit, Belgrade, Serbia) connected to an isometric transducer. The responses were expressed as contraction amplitude in grams.

Results were statistically evaluated by SPSS version 22 (IBM, Chicago, IL, USA) commercial software. Shapiro-Wilk test was used to estimate the data distribution. Data were compared among groups (separately for each sex) for each dose of ACh. One-way ANOVA with the Bonferroni post hoc test was used to compare data between groups. Comparison of male and female ileal contractions at the same ACh dose was performed by oneway ANOVA. The results are presented as the mean \pm SE. $P<0.05$ was considered significant.

\section{Results}

Statistical analysis showed no significant differences in the intensity of contractions between groups at the same ACh dose levels in both male (Fig. 1) and female (Fig. 2) ilea, 


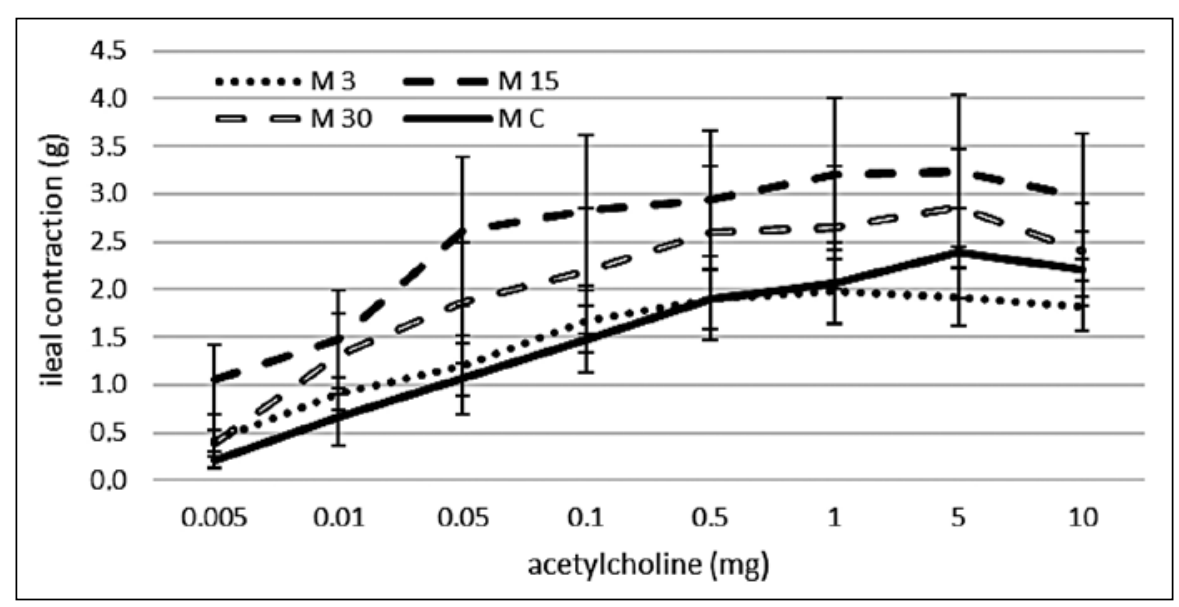

Figure 1. Contractions of male ilea ( $\mathrm{g}$ ) triggered by different amounts of acetylcholine. Organs originated from male rats that received chestnut extract as a feed additive for 90 days. Groups M 3, M 15 M 30 received feed containing 3, 15 and $30 \mathrm{~g}$ of chestnut extract per $\mathrm{kg}$, respectively. Group M C received no chestnut extract and served as a control group. There were 6 animals in each group.

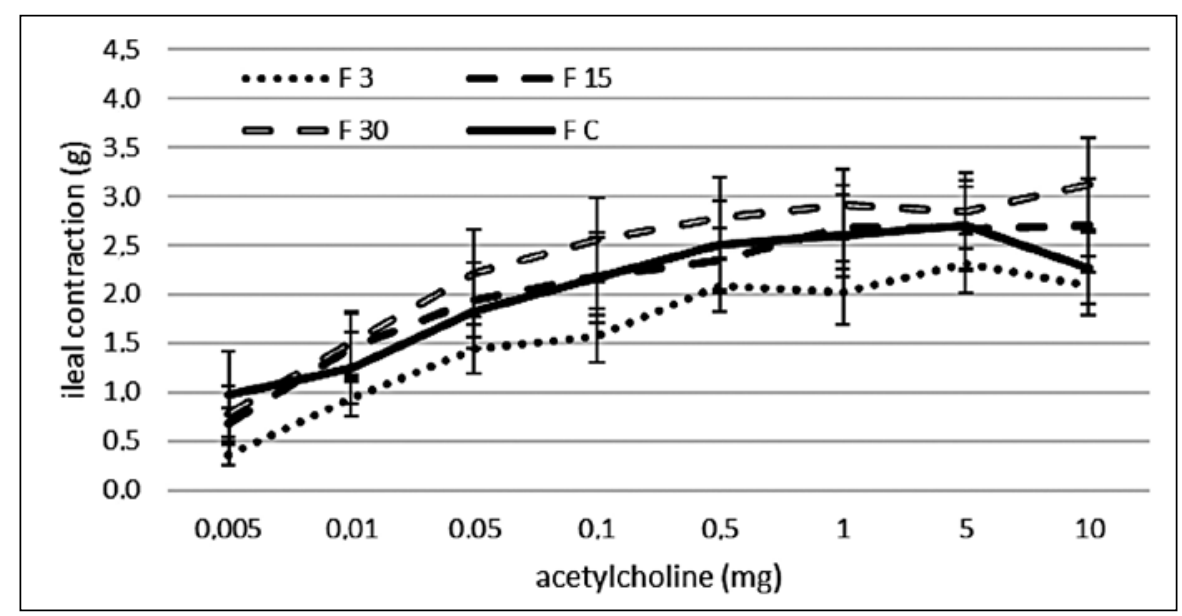

Fig. 2. Contractions of female ilea ( $\mathrm{g}$ ) triggered by different amounts of acetylcholine. Organs originated from female rats that received chestnut extract as a feed additive for 90 days. Groups F 3, F 15 and F 30 received feed containing 3,15 and $30 \mathrm{~g}$ of chestnut extract per $\mathrm{kg}$, respectively. Group F C received no chestnut extract and served as a control group. There were 6 animals in each group.

respectively. Additionally, comparison of the intensity of male and female ilea contractions at the same ACh dose showed no significant differences.

\section{Discussion}

Since the antispasmodic effect of tannins is well documented (Budriesi et al. 2010; Allem and Janbaz 2018), the goal of our study was to evaluate whether the long-term consumption of feed containing different amounts of chestnut extract influences the intestinal wall contraction ability. As shown by Figs 1 and 2, ileal contractions in all 
groups of both sexes showed dose-response steps to increasing ACh administration. These results suggest that previous long-term treatment with chestnut extract did not influence the contraction ability of the rat ileum.

Organs of both sexes were used to estimate eventual sex specific response to treatment with chestnut extract. The results suggest no influence of sex on the ileal contraction ability. It was reported that the antispasmodic effect of tannins is associated with their direct interaction with calcium channels such as nicotinic acetylcholine receptors, other cholinergic receptors, and calcium-activated chlorine channels (Budriesi et al. 2010; Namkung et al. 2010; Aleem and Janbaz 2018) in epithelial or smooth muscle cells, which are involved in the regulation of intestine motility. The results of this study showed that a long-term exposure to different and relatively high doses of tannins (higher than the doses used as a feed additive [Toral et al. 2011; Huang et al. 2018]) did not influence physiological mechanisms regulating ileal contractions. Although the contraction measurements in this study were performed immediately after the isolation of ilea from the body, treatment with chestnut extract had no observed impact on the ileal contraction ability under in vitro conditions. During the treatment, the experimental animals were clinically examined daily and showed no signs of lower feed intake or constipation. However, both anorexia and constipation may result from high amounts of hydrolysable tannins in feed (Gark et al. 1992). The higher doses that were used in this study (Table 1) could likely cause such adverse effects.

Interestingly, although non-significant, more intensive ileal contractions were observed in groups which received higher doses of chestnut extract (groups M 30, M 15, and F 30) in comparison to the groups treated with lower doses of chestnut extract and the control group. This trend was observed in male (Fig. 1) and female ilea (Fig. 2). If the chestnut extract at the used doses had caused antispasmodic activity, opposite results would have been observed. We believe that although high doses of chestnut extract were used, they were still too low to exhibit antispasmodic activity.

In conclusion, long-term administration of chestnut extract as a feed additive does not influence the intensity of ileal contractions triggered by ACh in laboratory rats.

To the best of our knowledge this is the first study which assesses the impact of chestnut extract as a feed additive on the intestinal contraction ability. This study was performed on laboratory rats and the results cannot be directly applied to other animal species. However, the activity of intestinal smooth muscle cells is regulated by calcium transport through membrane ion channels and by the vegetative nervous system (Sanders et al. 2012), a mechanism shared by most mammal species. Therefore, we believe that this study provides important information for the livestock industry as the results suggest that long-term use of chestnut extract as a feed additive has no adverse effect on intestinal motility. Further studies in target animal species are needed to completely confirm these suggestions.

\section{Acknowledgements}

This work was financially supported by The Slovenian Research Agency (grant number P4-0053) and Tanin Sevnica d.d. We thank Katarina Babnik and Boštjan Drolc for their technical assistance. The text was edited by American Journal Experts.

\section{References}

Aldred EM 2009: Pharmacology. Elsevier, Amsterdam, 352 p.

Aleem A, Janbaz KH 2018: Dual mechanisms of anti-muscarinic and $\mathrm{Ca}^{++}$antagonistic activities to validate the folkloric uses of Cyperus niveus Retz. as antispasmodic and antidiarrheal. J Ethnopharm 213: 138-148

Budriesi R, Ioan P, Micucci M, Micucci E, Limongelli V, Chiarini A 2010: Stop Fitan: antispasmodic effect of natural extract of chestnut wood in guinea pig ileum and proximal colon smooth muscle. J Med Food 13: 1104-1110

Garg SK, Makkar HP, Nagal KB, Sharma SK, Wadhwa DR, Singh B 1992:. Oak (Quercus incana) leaf poisoning in cattle. Vet Hum Toxicol 34: 161-164 
Huang Q, Liu X, Zhao G, Hu T, Wang Y 2018: Potential and challenges of tannins as an alternative to in-feed antibiotics for farm animal production. Anim Nutr 4: 137-150

Namkung W, Thiagarajah JR, Phuan PW, Verkman AS 2010: Inhibition of $\mathrm{Ca}^{2+}$-activated Cl- channels by gallotannins as a possible molecular basis for health benefits of red wine and green tea. FASEB J 24: 4178-4186

Piluzza G, Sulas L, Bullitta S 2013: Tannins in forage plants and their role in animal husbandry and environmental sustainability: a review. Grass Forage Sci 69: 32-48

Sanders KM, Koh SD, Ro S, Ward SM 2012: Regulation of gastrointestinal motility - insights from smooth muscle biology. Nat Rev Gastroenterol Hepatol 9: 633-645

Toral PG, Hervas G, Bichi E, Belenguer A, Frutos P 2011: Tannins as feed additives to modulate ruminal biohydrogenation: Effects on animal performance, milk fatty acid composition and ruminal fermentation in dairy ewes fed a diet containing sunflower oil. Anim Feed Sci Technol 164: 199-206 